\title{
Septicaemia in the elderly
}

\author{
J. W. MADDEN \\ M.B. B.S.
}

J. R. CROKER

M.R.C.P.

\author{
G. P. J. BEYNON \\ M.R.C.P.
}

Department of Geriatric Medicine, The Middlesex Hospital, Mortimer Street, London WIN 8AA

\begin{abstract}
Summary
A retrospective study was made of $\mathbf{4 4}$ elderly patients with bacteraemia treated in the period 1974-1980. Positive blood cultures in 5 cases were considered clinically insignificant. Twenty-six of the remaining 39 patients were found to have a Gram-negative bacteraemia, mainly associated with urinary tract infection. Abnormalities of liver function were common but 5 jaundiced patients with Gram-negative infection were shown to have stones in the common bile duct. The value of blood cultures as a diagnostic aid in the non-specifically ill elderly patient is emphasized.
\end{abstract}

\section{Introduction}

Wedgwood (1955) emphasized the difficulty of diagnosing infective endocarditis in the elderly. Elderly bacteraemic patients often present with illdefined symptoms and frequently have normal or subnormal temperatures.

Over the 6 months from September 1979 until February 1980, 91 pairs of blood cultures were taken (182 bottles). Twenty-five bottles grew an organism, and a positive culture was felt to be clinically significant in 22 cases $(12 \%)$. The preponderance of Gram-negative organisms in these cultures prompted the authors to review the records of their patients with bacteraemia during the years 1974 until 1980 . The distinction between bacteraemia and septicaemia is difficult and perhaps artificial in elderly patients. All the patients were symptomatic and they have been termed septicaemic.

\section{Patients and methods}

The Middlesex Hospital provides a geriatric admission service for acute problems in the elderly. Most patients over the age of 75 years with either acute medical, or mixed social and medical, problems are admitted under the geriatric firm. There are approximately 500 admissions yearly. Blood cultures were taken where clinically appropriate but particular indications included pyrexia, pneumoni⿱⺈ suspected urinary tract infection, sudden confusion or collapse, and suspected infective endocardit Each blood sample (approximately $10 \mathrm{ml}$ ) wos divided into 2 parts. One part was cultured aerobically in tryptone soya broth with $0.25 \%$ dextrose and the other anaerobically in thioglycollate $/ 0.1 \%$ aga Cultures were inspected daily. If they appeared cloudy a Gram stain was performed on a smear of the fluid and they were immediately sub-cultured 0 . samples were routinely sub-cultured after $7 \overrightarrow{\text { days }}$ incubation on to blood agar and MacConkeys medium and incubated for a further 7 days. The significance of any organism grown was determine clinically as far as possible. Biochemical and haomatological investigations were carried out in the majority of patients within $24 \mathrm{hr}$ of admission. Shoggk was defined as a state of peripheral circulatory collapse associated with a systolic BP below 90 mmHg.

\section{Results}

Positive blood cultures were obtained from patients but 5 patients were excluded because of the transient nature of their illness. The organisnas isolated from the remaining 39 patients are shown in Table 1. The average age of the patients was $\overline{81}$ years. There were 21 females and 18 males. Twentosix patients had a Gram-negative infection. In patients a single organism was found whilst bloge cultures grew more than one species of bacteria in ${ }^{3}$ patients.

\section{Gram-positive infection}

Most of these cases were associated with an easidy recognizable clinical condition (Table 2). Symptorns were generally non-specific. Ten patients had an altered level of consciousness. Rigors were nedt known to have occurred in any patient. Although 
TABLE 1. Organisms isolated from 39 patients with septicaemia

\begin{tabular}{lclc}
\hline \multicolumn{1}{c}{ Gram-positive species } & No. of cases & \multicolumn{1}{c}{ Gram-negative species } & No. of cases \\
\hline Streptococcus pneumoniae & 7 & $\begin{array}{l}\text { Escherichia coli } \\
\text { Proteus } \text { sp. }\end{array}$ & 17 \\
Streptococci-viridans & 3 & Bacteroides fragilis & 5 \\
$\beta$-haemolytic streptococci & 1 & Pseudomonas aeruginosa & 1 \\
Anaerobic streptococci & 1 & Proteus sp. and E. coli & 1 \\
Clostridium perfringens and micrococci & 1 & Klebsiella sp. and E. coli & 1 \\
$\quad$ Total & 13 & Total & 1 \\
\hline
\end{tabular}

TABLE 2. Diagnosis and culture in Gram-positive infection (13)

\begin{tabular}{|c|c|c|c|c|}
\hline \multirow{2}{*}{$\begin{array}{c}\text { Organism } \\
\text { Streptococcus pneumoniae }\end{array}$} & \multicolumn{2}{|c|}{ Underlying cause } & \multicolumn{2}{|l|}{ Clinical features } \\
\hline & $\begin{array}{l}\text { Pneumonia } \\
\text { Pneumonia + meningitis }\end{array}$ & $\begin{array}{l}6 \\
1\end{array}$ & $\begin{array}{l}\text { Signs of pneumonia } \\
\text { Radiological changes } \\
\text { Sputum available }\end{array}$ & $\begin{array}{l}7 \\
5 \\
4\end{array}$ \\
\hline Streptococci-viridans & Endocarditis & 3 & Embolic evidence & 2 \\
\hline$\beta$-haemolytic streptococci & Cellulitis of the leg & 1 & & \\
\hline Clostridium perfringens and micrococci & Sacral bedsore & 1 & & \\
\hline Anaerobic streptococci & Urinary tract infection & 1 & $\begin{array}{l}\text { Recent urinary retention and } \\
\text { catherization }\end{array}$ & \\
\hline
\end{tabular}

only one patient was apyrexial, the body temperature was below $38^{\circ} \mathrm{C}$ in 6 cases. Three patients were shocked, 2 of whom recovered.

In 3 cases both the total white cell and neutrophil cell counts were normal. Mild abnormalities of liver function were recorded in 4 patients. In 2 patients the blood urea was initially raised above $20 \mathrm{mmol} / 1$ but corrected with rehydration and both patients recovered. Two patients died from pneumonia despite appropriate antibiotic treatment (Table 6).

\section{Gram-negative infection}

Twenty-six patients had a Gram-negative infection (Table 1). Rigors and abdominal pain were more common in jaundiced than in non-jaundiced patients (Table 3). Eight patients were noted to have indwelling catheters.

Two patients were apyrexial and one other was frankly hypothermic $\left(28^{\circ} \mathrm{C}\right)$. Six were clinically shocked and 2 of these subsequently died. In 4 patients the white cell count was normal. A raised

TABLE 3. Symptoms in Gram-negative septicaemia

\begin{tabular}{lccc}
\hline \multicolumn{1}{c}{ Symptoms } & $\begin{array}{c}\text { Raised serum bilirubin } \\
\text { (7 cases) }\end{array}$ & $\begin{array}{c}\text { Other patients } \\
(19 \text { cases })\end{array}$ & Total \\
\hline Nausea and vomiting & 2 & 8 & 10 \\
Confusion & 2 & 6 & 8 \\
Rigors & 4 & 3 & 7 \\
Abdominal pain & 4 & 3 & 7 \\
Dysuria & 1 & 5 & 6 \\
Drowsiness & 0 & 4 & 4 \\
Diarrhoea & 2 & 2 & 4 \\
\hline
\end{tabular}


TABLE 4. Results in 7 patients with Gram-negative infection and raised from serum bilirubin levels

\begin{tabular}{lll}
\hline \multicolumn{1}{c}{ Blood culture } & \multicolumn{1}{c}{ Diagnosis } & \multicolumn{1}{c}{ Method of diagnosis } \\
\hline $\left.\begin{array}{l}\text { Klebsiella } \text { sp. } \\
\text { Escherichia coli }\end{array}\right\}$ & CBD stone & IVC and laparotomy \\
$\begin{array}{l}\text { E. coli } \\
\text { E. coli }\end{array}$ & CBD stone & IVC and laparotomy \\
$E$. coli & CBD stone & ERCP and laparotomy \\
$E$. coli & CBD stone & ERCP \\
$E$. coli & CBD stone & ERCP (died) \\
$E$. coli & ?CBD stone & Clinical \\
\hline
\end{tabular}

IVC=intravenous cholangiography; $\mathbf{E R C P}=$ endoscopic retrograde cholangio-pancreatography; $\mathrm{MSU}=$ mid-stream urine specimen; $\mathrm{CBD}=\mathrm{com}$ mon bile duct.

blood urea generally reflected dehydration and was $>20 \mathrm{mmol} / 1$ in 5 cases, 2 of whom died.

Abnormalities of liver function were present in 14 patients. Seven patients had a serum bilirubin above $15 \mathrm{mmol} / \mathrm{l}$. Common bile duct stones were demonstrated in 5 of these 7 patients (Table 4). In 4 the stones were successfully removed; in 3 , by formal surgery and in one case by endoscopic sphincterotomy. Endoscopic sphincterotomy failed to extract an enormous common bile duct stone (diameter
$3 \mathrm{~cm}$ ) in one patient who was unfit for surgery and who subsequently died. One patient fulfilled the criteria of Charcot's triad and was diagnosed" clinically as having cholangitis. This patient re covered and further investigation was not performede In the remaining patient liver function returned to normal after urinary tract infection was treated. $80 \overline{6}$ the 19 patients with normal serum bilirubin levels $?$ had moderate elevations of either serum alkatine phosphatase or serum transaminase concentrations.

TABLE 5. Results of blood and urine cultures (mid-stream urine and catheter specimen urine) in 19 patients with Gram-negative infection and normal serum bilirubin levels

\begin{tabular}{lll}
\hline \multicolumn{1}{c}{ Blood culture } & \multicolumn{1}{c}{ Urine } & Numbers \\
\hline Escherichia coli & E. coli (CSU) & 2 \\
E. coli & Mixed growth (CSU) & 1 \\
Proteus sp. & Proteus (CSU) & 2 \\
Proteus sp. & Mixed growth (CSU) & 2 \\
Pseudomonas sp. & Mixed growth (CSU) & 1 \\
E. coli & Coliforms (MSU) & 5 \\
E. coli & Mixed growth (MSU) & 1 \\
E. coli & No growth 100 WBC (MSU) & 1 \\
E. coli/Proteus sp. & Coliform (MSU) & 1 \\
Proteus sp. & Mixed growth (MSU) & 1 \\
Bacteroides sp. & Mixed growth (MSU) & 1 \\
E. coli & No record & 1 \\
& Total & 19 \\
\hline
\end{tabular}

$\mathrm{CSU}=$ catheter specimen of urine; $\mathrm{MSU}=$ mid-stream urine specimen. 
TABLE 6. Details of patients who died

\begin{tabular}{lll}
\hline \multicolumn{1}{c}{ Type } & \multicolumn{1}{c}{ Source } & \multicolumn{1}{c}{ Comments } \\
\hline Gram-positive & Pneumonia & Shock, hypothermia, malnourished \\
Gram-positive & Pneumonia & Recent resection of Dukes 'C' carcinoma of rectum \\
Gram-negative & CBD stone & Impacted stone \\
Gram-negative & Urinary tract & Uraemia. Catheterized 6 days \\
Gram-negative & Bowel & Shock. Perforated bowel \\
Gram-negative & Urinary tract & Uraemia, hypotension, hypothermia, diabetic ketoacidosis \\
\hline
\end{tabular}

$\mathrm{CBD}=$ common bile duct.

The source of infection in patients without elevation of serum bilirubin levels was more difficult to establish. Urine was obtained for culture before antibiotic therapy was commenced in $\mathbf{1 8}$ of the 19 patients (Table 5). Two patients were catheterized during the $24 \mathrm{hr}$ preceding symptoms. A pure growth of organism was obtained in 10 cases (4 of 8 with indwelling catheters). It is the practice of the Bacteriology Department not to sub-group coliform organisms in urine specimens. Comparison of the antibiotic sensitivities of blood and urine cultures in these cases, however, supported a diagnosis of urinary tract infection. A mixed growth of organisms was found in the remaining 8 patients ( 4 with indwelling catheters), 2 of whom had significant urinary symptoms. There was therefore reasonable evidence to support a diagnosis of underlying urinary tract infection in 12 patients.

Four patients with Gram-negative infection died with septicaemia (Table 6).

\section{Antibiotic therapy}

Except for patients with suspected endocarditis, all patients were treated while blood culture results were awaited. The choice of antibiotic was governed by the clinical presentation. Where Gram-negative infection was suspected, as in cholangitis or urinary tract infection, ampicillin was the commonest antibiotic used, frequently in combination with gentamicin or metronidazole. Gentamicin dosage was controlled by serum levels. Corticosteroids were used in one shocked patient, who recovered.

\section{Discussion}

The incidence of bacteraemia is rising in hospital practice (Altemeier, Todd and Inge, 1967) and is owing in part to the increasing contribution from the geriatric population. More blood cultures are now performed because of increased awareness, and this partly accounts for increasing diagnosis (Crowley, 1970). Gram-negative bacteraemia is now more common and Escherichia coli is the predominant offending organism (Geddes, 1978). In this series, two-thirds of cases were due to Gramnegative organisms.

Disease in the elderly commonly presents nonspecifically, and bacteraemia is no exception to this rule. Altogether 22 of the 39 patients presented with confusion or drowsiness. Although the clinical presentation may not distinguish between Grampositive and Gram-negative infection (Winslow et al., 1973), in this series rigors and jaundice were more common in Gram-negative infection and the source of infection was more obvious in patients with Gram-positive bacteraemia (Table 2).

Abnormalities of liver function were common and present in 18 cases (4 Gram-positive, 14 Gramnegative), although a raised serum alkaline phosphatase was not always proved to be of hepatic origin. Elevation of serum transaminase concentration is thought to be the rule in septicaemia (Geddes, 1978) but was found in only 7 cases. The serum bilirubin concentration was raised in 9 patients, 7 of whom had Gram-negative infections. Common bile duct stones were subsequently demonstrated in 5 of these 7 cases and suspected in one other. Although a selective defect in excretion of conjugated bilirubin has been described in severe infection (Miller et al., 1976) it is believed that the presence of jaundice with Gramnegative septicaemia necessitates exclusion of biliary stones even when clinical features of cholangitis are lacking. Gall stones occur in about $30 \%$ of the over-70 age group (Bateson and Bouchier, 1975), and although frequently 'silent' in the elderly (Sato and Matsushiro, 1974) they may present suddenly with septicaemic shock (Faber et al., 1978). Grey scale ultrasonography readily demonstrates intrahepatic duct dilatation and accurately detects calculi in the gall bladder. Stones in the bile duct, however, are more difficult to see and may cause jaundice without biliary dilatation (Vallon, Lees and Cotton, 1979). Endoscopic cholangiography is accurate, and 
non-operative removal of bile duct stones by duodenoscopic sphincterotomy is a major advance in elderly and high risk patients (Cotton, 1980).

Significant underlying disease may be present in $95 \%$ of cases of Gram-negative bacteraemia (McHenry, Martin and Wellman, 1962) and multiple pathology was common amongst most of the present patients. Increasing surgery and instrumentation contribute to infection. The urinary tract may be the source of Gram-negative bacteraemia in over $50 \%$ of patients (Altemeier et al., 1967). In this series, the urinary tract was the proved source of infection in 11 cases (10 with Gram-negative infection) and the suspected source in 6 others. Eight of the patients with Gram-negative bacteraemia had indwelling catheters at presentation. In 2 cases, catheters had been inserted in the $24 \mathrm{hr}$ before illness and in a third patient 6 days earlier.

Blood cultures both confirm a diagnosis of bacteraemia and provide a rational basis for antibiotic therapy. In many cases the results of blood culture led to changes in antibiotic treatment, but this influence was difficult to assess retrospectively. In 3 patients a positive blood culture confirmed a diagnosis of suspected endocarditis. Blood cultures are of value in the management of pneumonia since sputum, even when available, may not accurately reflect the pathogenic organism growing in the lung (Barrett-Connor, 1971). Many elderly patients present non-specifically and a high index of clinical suspicion is required to make a diagnosis of bacteraemia. In particular, a history of recent catheterization made the authors suspect bacteraemia, and the presence of jaundice with a positive blood culture made them suspect cholangitis.

Although blood cultures are of value in confirming a diagnosis of bacteraemia, suspected cases require early active treatment. Intravenous antibiotics should be given whilst blood culture results are awaited; the choice of antibiotic being governed by the clinical situation. Mortality in septicaemia may rise to 50\% (Altemeier et al., 1967) and may reach $90 \%$ in the presence of shock (Winslow et al., 1973). There were 6 deaths in the patients in this study (mortality $15 \%$ ) but mortality rose to $33 \%$ in those patients who were shocked $(23 \%)$. Catecholamines (Tinker, 1979) have been used in treating septicaemia complicated by shock, but the value of steroids is based on uncontrolled haemodynamic studiōs (Schumer, 1976). The outcome of septicaemia in this series was related to the general health of the patient and to the presence of underlying disease. All जo patients who died had serious illness (Table 6). Twख patients who were found to be hypothermic on admission both died.

\section{Acknowledgments}

The authors thank Dr M. Shiels of the Microbiolog Department for advice, and Dr J. Wedgwood for allowing us to study his patients.

\section{References}

Altemeier, W.A., Todo, J.C. \& INGe, W.W. (1967) Gran, negative septicaemia: a growing threat. Annals of Surgery $166,530$.

BARRETT-CONNOR, E. (1971) The non-value of sputung culture in the diagnosis of pneumococcal pneumonia American Review of Respiratory Diseases, 103, 845.

Bateson, M.C. \& Bouchier, I.A.D. (1975) Prevalence of gall stones in Dundee: a necropsy study. British Medic Journal, 4, 427.

Cotton, P.B. (1980) Non-operative removal of bile dust stones by endoscopic sphincterotomy. British Journal 영 Surgery, 67, 1.

Crowley, N. (1970) Some bacteraemias encountered hospital practice. Journal of Clinical Pathology, 23, 16 $\overrightarrow{0}$

FABer, R.G., IBRAHIM, S.Z., ThOMAS, D.M., BEY G.P.J. \& LE QUESNE, L.P. (1978) Gall stone disease \$resenting as septicaemic shock. British Journal of Surserv 65,101 .

GEDDES, A.M. (1978) Use of antibiotics: septicaemi British Medical Journal, 2, 181.

McHenry, M.D., Martin, W.J. \& Wellman, W.E. (1969) Bacteraemia due to Gram-negative bacilli. Annals of Internal Medicine, 52, 207.

Miller, D.J., Keeton, G.R., Webber, B.L. \& SAUnders, S. (1976) Jaundice in severe bacterial infection. Gastroenter $\bar{\theta}$ $\log y, 71,94$.

Sato, T. \& Matsushiro, T. (1974) Surgical indications 苨 patients with silent gall stones. American Journal of Surgery, 128, 368.

SCHumER, W. (1976) Steroids in the treatment of clinica. septic shock. Annals of Surgery, 184, 333.

TINKER, J. (1979) A pharmacological approach to the treatment of shock. British Journal of Hospital Medicinie 22, 261.

Vallon, A.G., Less, W.R. \& Cotton, P.B. (1979) Gre辵 scale ultrasonography in cholestatic jaundice. Gut, 20, 5Q

WEDGWOOD, J. (1955) Early diagnosis of subacute bacteriậ endocarditis. Lancet, ii, 1058.

Winslow, F.J., Loeb, H.S., Rahimtoola, S.H., Kamath, \& GunNar, R.M. (1973) Hemodynamic studies and resulto of therapy in 50 patients with bacteremic shock. American Journal of Medicine, 54, 421. 